\title{
The VEGF pathway and the AKT/mTOR/p70S6KI signalling pathway in human epithelial ovarian cancer
}

\author{
XB Trinh',2, WAA Tjalma², PB Vermeulen', G Van den Eynden', I Van der Auwera', SJ Van Laere', \\ J Helleman', EMJJ Berns', LY Dirix' and PA van Dam*,I
}

'Translational Cancer Research Group Antwerp, St Augustinus Hospital, Antwerp, Belgium; ' 2 epartment of Gynaecological Oncology, Antwerp University Hospital, Antwerp, Belgium; ${ }^{3}$ Department of Medical Oncology, Erasmus MClJosephine Nefkens Institute, Rotterdam, The Netherlands

\begin{abstract}
Vascular endothelial growth factor (VEGF)-A inhibitors exhibit unseen high responses and toxicity in recurrent epithelial ovarian cancer suggesting an important role for the VEGF/VEGFR pathway. We studied the correlation of VEGF signalling and AKT/mTOR signalling. Using a tissue microarray of clinical samples $(N=86)$, tumour cell immunohistochemical staining of AKT/mTOR downstream targets, pS6 and p4E-BPI, together with tumour cell staining of VEGF-A and pVEGFR2 were semi-quantified. A correlation was found between the marker for VEGFR2 activation (pVEGFR2) and a downstream target of AKT/mTOR signalling $(\mathrm{pS6})(R=0.29$; $P=0.002)$. Additional gene expression analysis in an independent cDNA microarray dataset $(N=24)$ showed a negative correlation $(R=-0.73, P<0.000 I)$ between the RPS6 and the VEGFR2 gene, which is consistent as the gene expression and phosphorylation of $\mathrm{S} 6$ is inversely regulated. An activated tumour cell VEGFR2/AKT/mTOR pathway was associated with increased incidence of ascites $\left(\chi^{2}, P=0.002\right)$ and reduced overall survival of cisplatin-taxane-based patients with serous histology $(N=32$, log-rank test, $P=0.04)$. These data propose that VEGF-A signalling acts on tumour cells as a stimulator of the AKT/mTOR pathway. Although VEGF-A inhibitors are classified as anti-angiogenic drugs, these data suggest that the working mechanism has an important additional modality of targeting the tumour cells directly.
\end{abstract}

British Journal of Cancer (2009) 100,971-978. doi: 10.1038/sj.bjc.660492 I www.bjcancer.com

Published online 24 February 2009

(c) 2009 Cancer Research UK

Keywords: mTOR; VEGFR2; angiogenesis; autocrine; VEGF-A; ovarian cancer

In the Western world, ovarian cancer has the highest mortality rate among gynaecological malignancies (Jemal et al, 2008). There is an unmet need for novel targeted therapies that can improve current treatment outcomes (Kelland, 2005). In early clinical studies, remarkable activity of single agents targeting VEGF-A has been observed (Martin and Schilder, 2007). The working mechanism of these anti-VEGF-A inhibitors is believed to be inhibition of angiogenesis as VEGF-A is a critical and major step in angiogenesis (Ferrara, 2004, 2005). As single agent, bevacizumab, the monoclonal humanised antibody against VEGF-A, yielded response rates of 15.9 and $21.0 \%$ in refractory ovarian cancer patients in phase II trials and is currently the subject of phase III trials (Burger, 2007; Burger et al, 2007; Cannistra et al, 2007). In comparison, although it is difficult to compare different phase II trials, the observed response rate with single-agent bevacizumab was only $3.3 \%$ in metastatic colorectal cancer (Giantonio et al, 2007). In a metastatic renal cell cancer phase II trial, the response of single-agent high-dose bevacizumab was found to be $10.3 \%$, whereas in metastatic breast cancer the total objective response rate was 6.7\% (Cobleigh et al, 2003; Yang et al, 2003). In addition to its apparently different antitumoural effect, the highest

*Correspondence: Dr PA van Dam, St Augustinus Hospital, Oosterveldlaan 24, 2610 Wilrijk, Antwerp, Belgium; E-mail: peter.vandam@gza.be Received 9 October 2008; revised 9 January 2009; accepted 12 January 2009; published online 24 February 2009 incidence of gastrointestinal perforations (6\%) is observed in ovarian cancer compared with the overall incidence of $1.7 \%$ in patients who receive bevacizumab for malignancies (Badgwell et al, 2008). In one phase II trial, the incidence was even found to be $11 \%$ (Cannistra et al, 2007).

The distinct response rates and toxicity observed indicate that VEGF-A signalling may have different mechanisms depending on the tumour type studied and also that there is a particular prominent role for VEGF-A in ovarian cancer. Therefore, further research of VEGF-A mechanisms in ovarian cancer is warranted. Several reports have identified the VEGFR2, also known as KDR, on epithelial tumour cells, including its localisation in ovarian cancer cells (Masood et al, 2001; Stewart et al, 2003; Inan et al, 2006). In vitro studies have suggested an autocrine growth factor function of VEGF-A/VEGFR2 signalling (Masood et al, 2001; Inan et al, 2006). However, the clinical importance of these non-endothelial VEGF receptors remains elusive. In this study, we assessed the VEGF-A/VEGFR2 pathway in human ovarian carcinoma tissue in terms of VEGF expression, VEGFR2 expression, and its activation status. As VEGFR2 exerts its function through the PI3K/Akt/mTOR signalling pathway in endothelial cells (Figure 1), the activation status of VEGFR2 was studied by determining phosphorylation of VEGFR2 itself together with phosphorylation of downstream targets of mTOR. In addition, the association of VEGFA, VEGFR2 and their activation status with clinical outcome were established. 


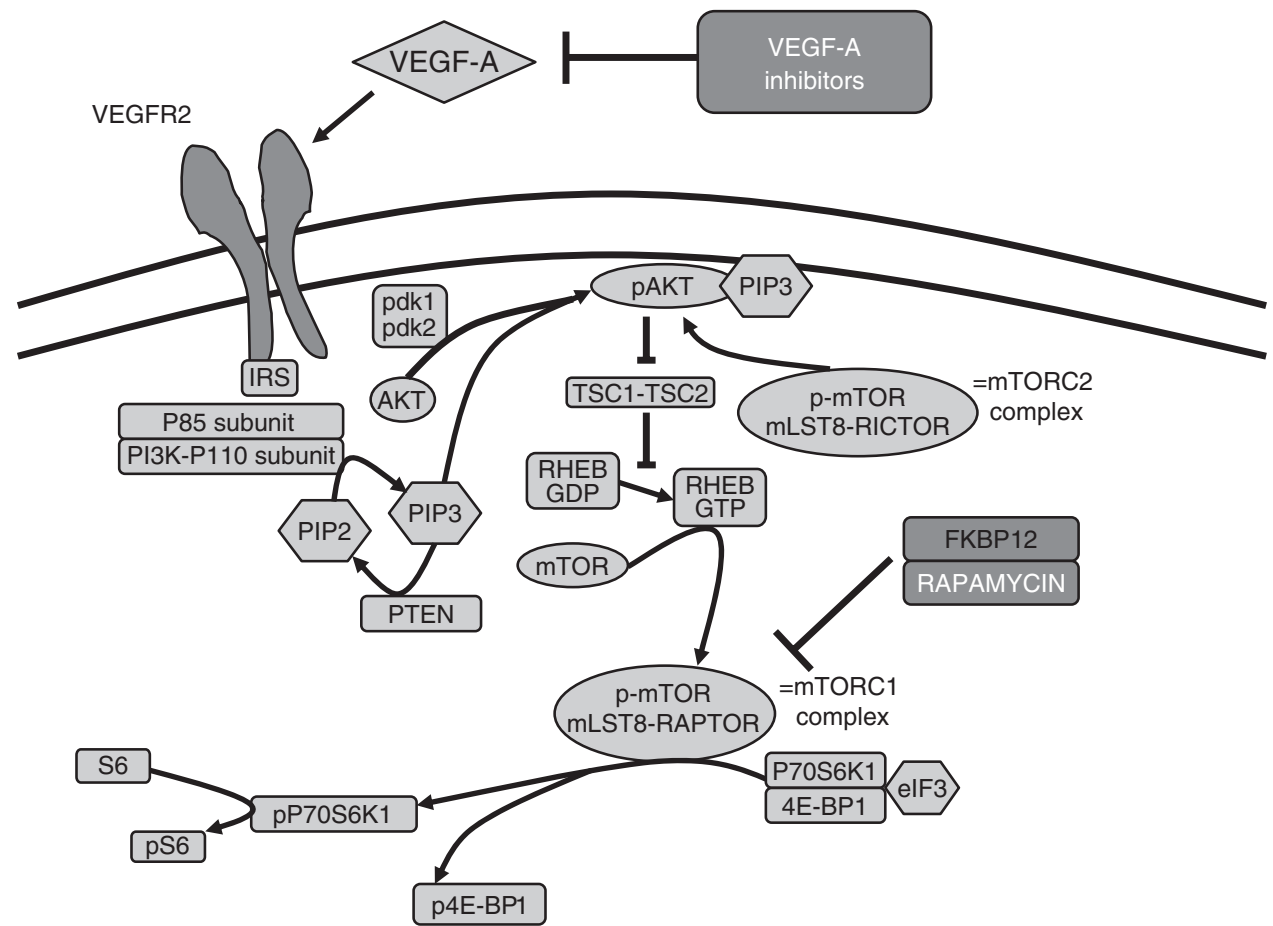

Figure I In endothelial cells, after activation, VEGFR2 is able to stimulate the AKT/mTOR signalling pathway through PI3K. More downstream 4E-BP I, P70S6KI and S6 are phosphorylated. The mTORC2 complex (insensitive to rapamycin/FKBPI2) has been shown to be an activator of AKT. The mTORCI complex is sensitive to rapamycin (sirolimus) or derivates (everolimus, temsirolimus and deforolimus).

\section{MATERIALS AND METHODS}

\section{Patient and tissue selection}

Formalin-fixed paraffin-embedded (FFPE) epithelial ovarian cancer tissues were collected retrospectively from 1999 to 2004 in the St Augustinus hospital in Belgium using pathology archives. The local IRB reviewed and approved the study. Clinical parameters were obtained from patients' medical files, that is, FIGO stage, grade, age at the time of diagnosis, date and type of surgery, histology, presence of ascites and treatment modalities. Remaining tumour load after debulking, which was not consistently recorded, was not included. The patients' characteristics are listed in Table 1 and the biopsy characteristics in Figure 2. Median age of the patients was 61.6 years (27-92 years). Twelve patients did not receive systemic treatment. The majority received platinum in combination with taxane chemotherapy $(N=33)$. Other treatments included carboplatinum monotherapy $(N=1)$, carboplatinum cyclofosfamide $(N=5)$ and cyclophosphamide-adriamycincisplatin $(N=2)$.

Tissue samples from 89 patients were included on the tissue microarray (TMA). Primary untreated ovarian lesions were available in 86 out of 89 patients, whereas in the remaining three subjects, only an untreated peritoneal biopsy was available (Figure 2). In 49 out of 89 patients, metastatic (peritoneal or omental) lesions were surgically removed at the same time by laparotomy, either during primary debulking or during interval debulking. Tissues were also collected from patients who had neoadjuvant chemotherapy before debulking surgery. In these patients, (16 out of 89) there was tissue from baseline biopsy and tissue from debulking surgery, which were all performed after three cycles of taxane-platinum chemotherapy.

\section{Tissue microarray}

Using the Beecher Instruments Tissue Arrayer (Beecher Instruments, Silver Springs, MD, USA) a TMA was constructed.
Haematoxylin eosin (HE) staining for each paraffin block was reviewed and three representative areas were selected on the HE slide. From each paraffin block, three sample cores were taken, each of which corresponded to the earlier selected areas on the HE slide. Five- $\mu \mathrm{m}$ slides were cut from the TMA for immunohistochemical staining.

\section{Immunohistochemistry}

Immunohistochemistry was performed on consecutive slides for pS6, p4E-BP1, pVEGFR2(Tyr996) (Cell Signalling Technologies, Beverly, MA, USA; \#2211, \#2474, \#9451), pVEGFR2(Tyr951) (Santa Cruz Biotechnologies, Santa Cruz, CA, USA; \#sc-16628-R) and VEGF-A (Dako Corp., Glostrup, Denmark, VG1clone M7273). After deparaffinisation and rehydration, antigen retrieval was performed using a citrate buffer (pH6.0) (pS6, p4E-BP1, VEGF-A) or an EDTA buffer (pH9.0) (pVEGFR2) at sub-boiling temperature for $30 \mathrm{~min}$. After $30 \mathrm{~min}$ of cooling on bench top, sections were treated with $1 \% \mathrm{H}_{2} \mathrm{O}_{2}$ and then incubated with the primary antibodies for $1 \mathrm{~h}$ at room temperature at $1: 200$ dilution for pS6, $1: 50$ for p4E-BP1, 1:100 for pVEGFR2(Tyr996) and 1:150 for pVEGFR2(Tyr951). Binding of antibodies was visualised using Envisionplus dual link system (Dako Corp.) and 3,3-diaminobenzidine for $10 \mathrm{~min}$. Sections were counterstained with Mayer's haematoxylin. For the VEGF-A staining, a 1:800 dilution with 1-h incubation was used with the Catalyzed Signal Amplification kit (CSA kit Dako Corp.). Ki67 staining was performed as described earlier (Van den Eynden et al, 2007). A breast carcinoma and melanoma (for VEGF-A, pVEGFR2), and a prostate cancer specimen (for pS6, p4E-BP1) served as positive controls. Negative controls were run by omitting the primary antibody. All the staining was done on the Dako autostainer (Dako Corp.).

\section{Quantifictation of the staining}

The $H$-score, that is, the percentage of cells staining positive multiplied by an intensity score $(0-3)$, was used for 
Table I Summary of clinical data $(N=89$ patients)

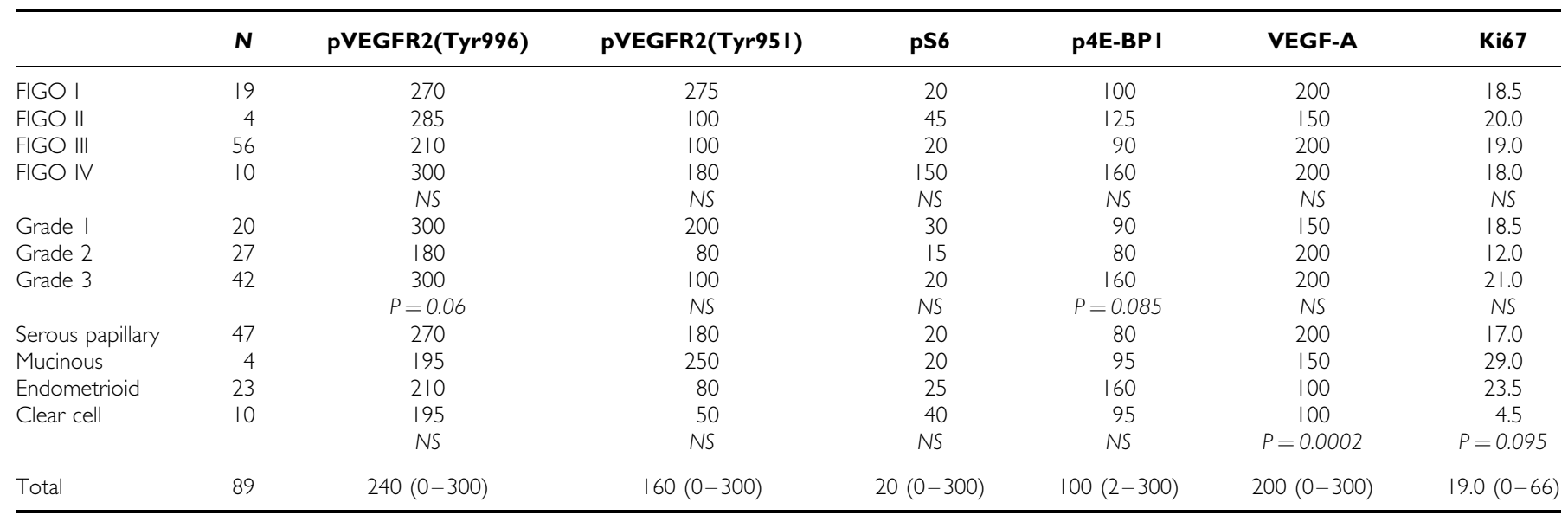

Median $H$-scores are reported. $P$-values (Kruskal-Wallis tests) are reported in case of significance $(P<0.05)$ or in case of trend $(P<0.10)$. After Bonferroni correction for multiple testing $P<0.008$ was considered significant.

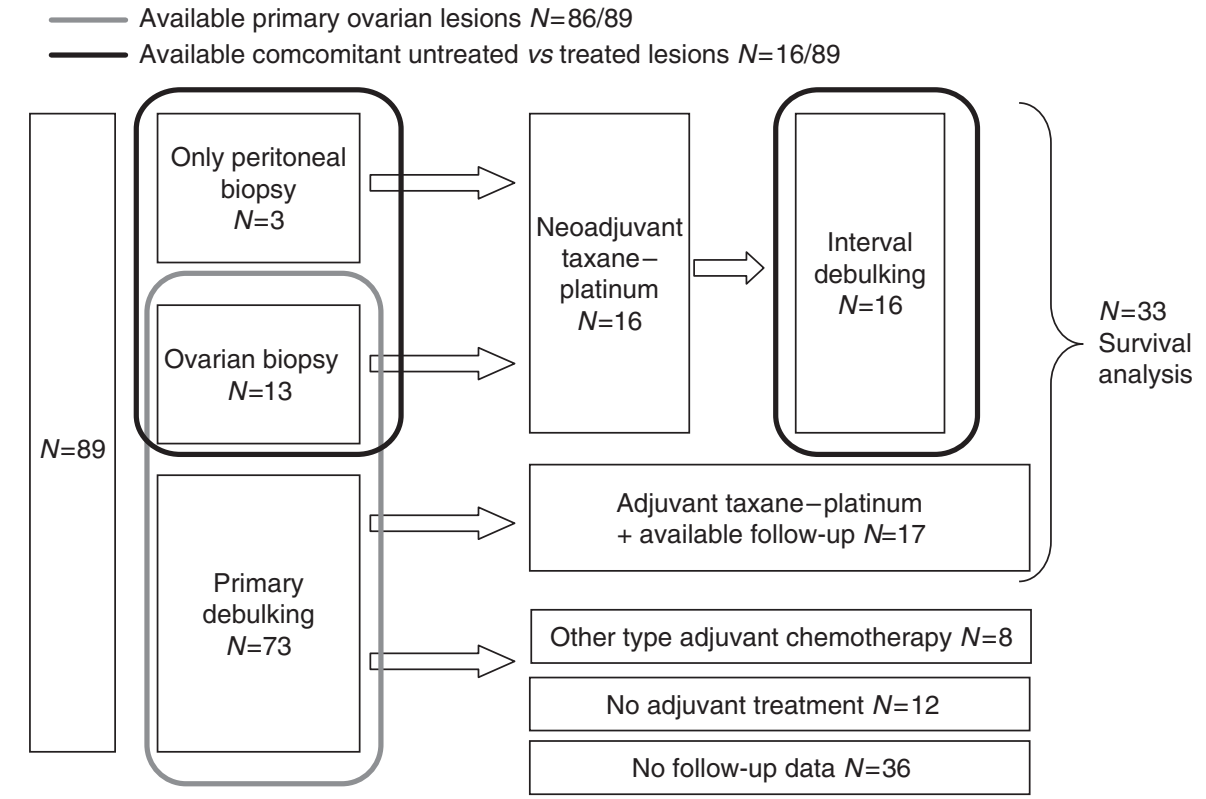

Figure 2 Schematic overview of patients included in the study.

semi-quantification of the staining on the tumour cells (Rojo et al, 2007). The semi-quantification was performed by two independent observers who were blinded from clinical data. As three cores were taken from a single paraffin block, the average $H$-score over the three cores was used for further analysis. Ki67 staining was quantified with a proliferation index (number of Ki67 positive proliferating tumour cells per 100 tumour cells) using 200 counted cells per tissue core.

\section{Statistical and survival analysis}

All statistical analyses were performed using SPSS 13.0 (SPSS Inc., Chicago, IL, USA) and GraphPad Prism 3.03 (GraphPad Software, Inc., La Jolla, CA, USA) statistical packages. Marker correlation analysis of $\mathrm{H}$-scores was performed using the Spearman correlation coefficient. For survival analysis, only those patients who had taxane-platinum first line chemotherapy and serous papillary histology were selected $(N=32)$. For this, the expression of pVEGFR2 and pS6 was dichotomised in high or low category using the median expression as a cutoff value. Wilcoxon matched paired testing was performed on the $\mathrm{H}$-scores if primary ovarian tissue as well as a metastatic lesion was available from the same patient, at the same time of surgical excision $(N=49)$. Similarly, Wilcoxon matched paired testing was conducted if the tissue, before chemotherapeutical treatment and after neoadjuvant taxane-platinum chemotherapy, was available from the same patient $(N=16)$.

\section{In silico gene expression analysis}

Normalised gene expression data was derived from a molecular profiling study described earlier, including 24 independent untreated primary ovarian cancer lesions, using $18 \mathrm{~K}$ cDNA microarray (Helleman et al, 2006; Erasmus MC, Rotterdam, The Netherlands). The expression values of RPS6 (coding for S6 protein), EIF4EBP1 (coding for $4 \mathrm{E}-\mathrm{BP} 1$ protein) and VEGFR2/KDR were analysed for correlation studies. The mean of duplicate analyses was used. In addition, gene expressions for RPS6 and EIF4EBP1 were derived from a publicly available gene expression 


\section{A}
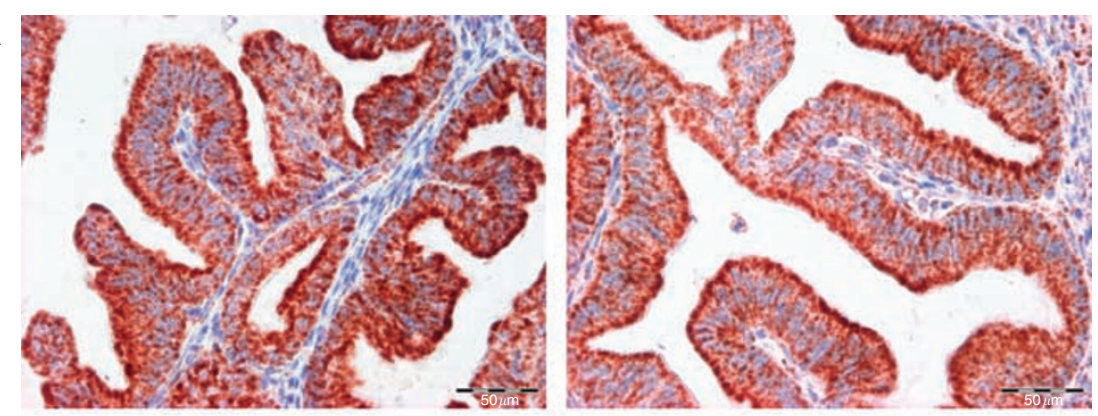

B
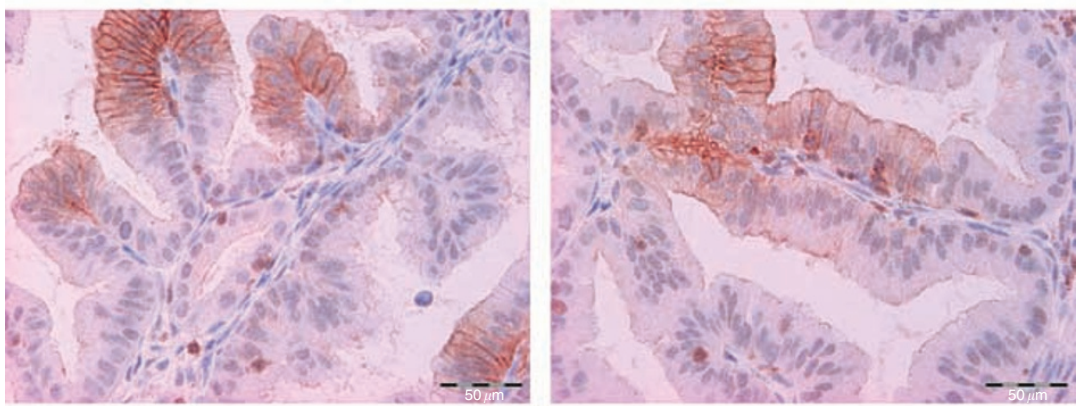

C
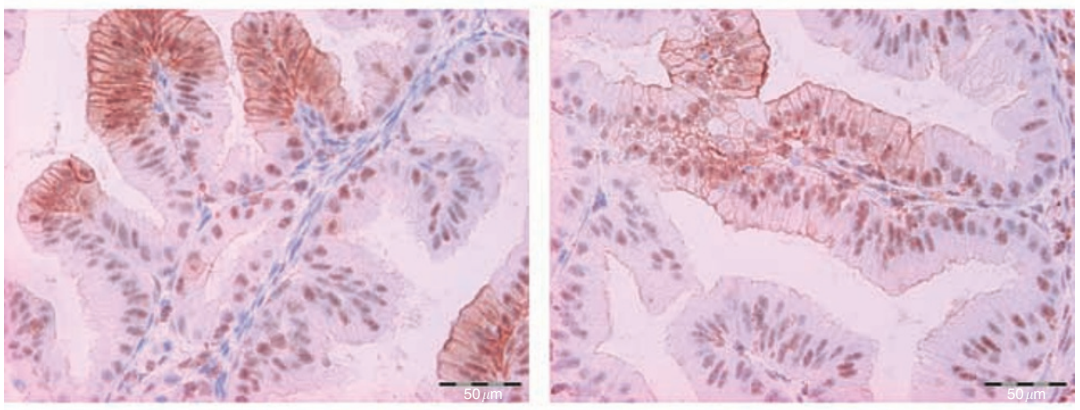

D
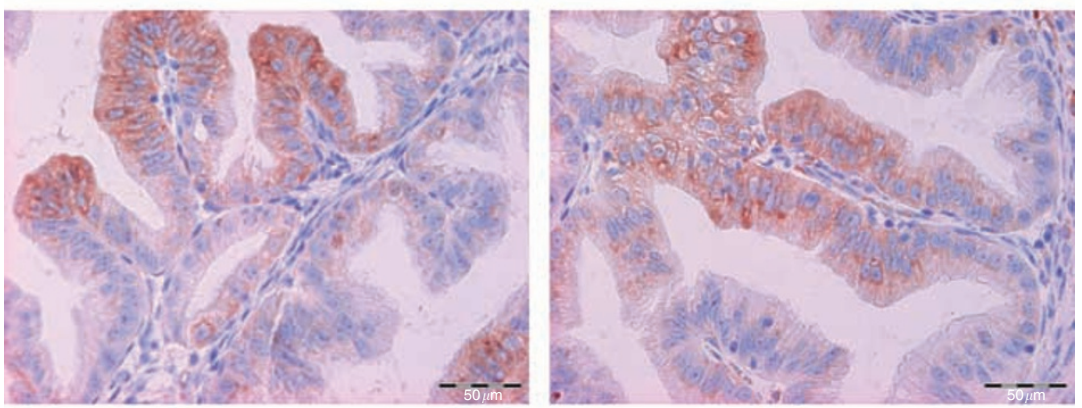

E
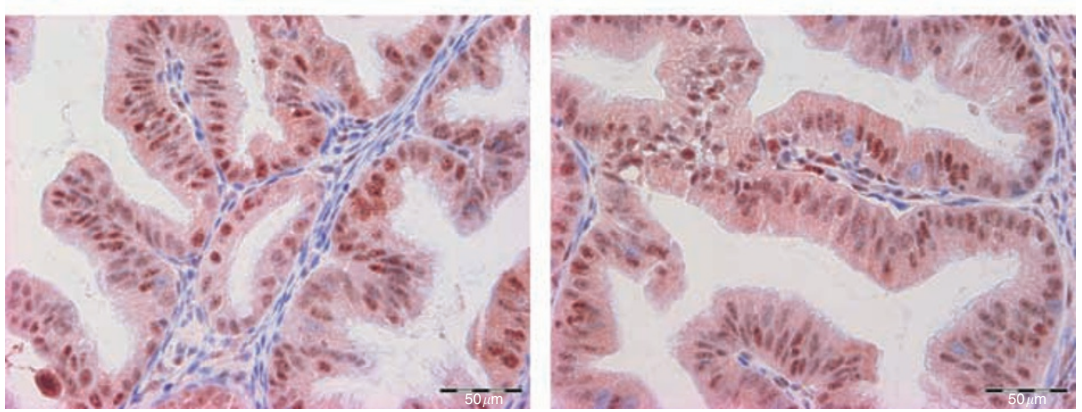

Figure 3 (A-E) Serial slides with immunohistochemical staining for pVEGFR2(Tyr95I) (A), pVEGFR2(Tyr996) (B), pS6 (C), p4E-BPI (D), VEGF-A (E) of an ovarian cancer specimen. Note that there were locally places in which there was more prominent staining for pVEGFR2 with a concomitant expression of the ribosomal protein pS6.

omnibus dataset of Mus musculus prostate samples before and after (12 and $48 \mathrm{~h}$ ) mTOR inhibition with the RAD001 compound. These samples were processed using Affymetrix GeneChip Mouse
Expression Set 430 Array MOE430A (Affymetrix Inc., Santa Clara, CA, USA). Microarrays were background adjusted, normalised, summarised and ${ }^{2} \log$ transformed according to GC Robust 


\begin{tabular}{|c|c|c|c|c|c|}
\hline $\begin{array}{c}R \text { Spearman } \\
\text { Correlation } \\
\text { Coefficient }\end{array}$ & VEGF-A & $\begin{array}{c}\text { pVEGFR2 } \\
(996)\end{array}$ & $\begin{array}{c}\text { pVEGFR2 } \\
(951)\end{array}$ & pS6 & p4E-BP1 \\
\hline VEGF-A & 1 & 0.25 & 0.29 & NS & NS \\
\hline $\begin{array}{c}\text { pVEGFR2 } \\
(996)\end{array}$ & 0.25 & 1 & 0.70 & 0.29 & 0.18 \\
\hline $\begin{array}{c}\text { pVEGFR2 } \\
(951)\end{array}$ & 0.29 & 0.70 & 1 & 0.20 & 0.20 \\
\hline pS6 & NS & 0.29 & 0.21 & 1 & 0.21 \\
\hline p4E-BP1 & NS & 0.18 & 0.20 & 0.21 & 1 \\
\hline \multicolumn{7}{|c|}{} & NS & NS & NS & NS & 0.22 \\
\hline Ki67 &
\end{tabular}

\begin{tabular}{|l|l|}
\hline & Perfect correlation \\
\hline & Correlation with $P<0.01$ \\
\hline & Correlation with $P<0.05$ \\
\hline & No significant correlation \\
\hline
\end{tabular}

Figure 4 A summary of immunohistochemical correlations.

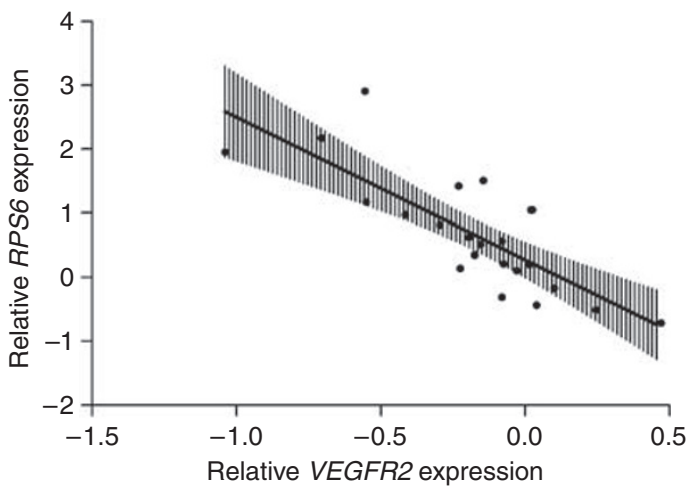

Figure 5 The ${ }^{2} \log$ relative gene expression correlations using an independent dataset of epithelial ovarian cancer samples. The RPS6 gene was significantly well correlated with the relative expression of VEGFR2. (vertical bars show 95\% Cl).

Miroarray method. Nine probe set ID's were available for analysis of the RPS6 gene and two were available for EIF4EBP1 gene. Samples were divided into three groups: placebo treated $(N=18)$, $12 \mathrm{~h}$ RAD001 treated $(N=11)$ and $48 \mathrm{~h}$ RAD001 treated $(N=9)$. One-way ANOVA tests with additional Student-Newman-Keul's post test (if $P<0.05$ ) were performed.

\section{RESULTS}

\section{Immunostaining and marker correlative studies}

An example of staining of pVEGFR2(Tyr996), pVEGFR2(Tyr951) pS6, p4E-BP1 and VEGF-A is shown in Figure 3. A summary of the staining $H$-scores is listed in Table 1 , whereas the correlations are presented in Figure 4. VEGF-A expression is most prevalent in serous papillary tumours $(P=0.0002)$. The staining of the VEGFR2 phosphorylated at tyrosine residue 996 or 951 was found to be cytoplasmic, nuclear and/or membranous. There was a correlation
( $R=0.70, P<0.00001$ ) between both pVEGFR 2 staining, namely the VEGFR2 phosphorylated at tyrosine residue 951 and that phosphorylated at tyrosine residue 996, using different antibodies that were raised to detect different epitopes. The staining patterns for pS6, p4E-BP1 and nuclear ki67 staining were similar to the reports described earlier (Van den Eynden et al, 2005; Castellvi et al, 2006; Rojo et al, 2007). A predominantly often granular staining of VEGF-A was observed in the tumour cell cytoplasm as well in the stromal compartment of the tumour.

For correlations, (Figure 4) primary untreated ovarian lesions $(N=86)$ were included. Correlations between pVEGFR2(Tyr996) and downstream markers for the AKT/mTOR signalling pathway (pS6 and p4E-BP1) were found for pVEGFR2(Tyr996) and pS6 $(R=0.29, P=0.002)$, and also for pVEGFR2(Tyr996) and p4E-BP1 $(R=0.18, P=0.05)$.

When studying only metastatic lesions $(N=59)$, the correlation of pVEGFR2(Tyr996)-pS6 was even more pronounced $(R=0.44$, $P<0.0001)$, whereas there was no correlation between pVEGFR2 (Tyr996)-p4E-BP1 $(R=0.12 P=0.25)$. Interestingly, the expression of pVEGFR2(Tyr996) was found to be significantly lower in metastatic lesions when compared with primary ovarian lesions surgically removed at the same time (median $H$-score $=90$, range $(0-300)$ vs 150 , range $(0-300) ; P=0.01, N=49)$. The expression of pS6 and p4E-BP1 was not significantly different.

The tissue microarray also contained samples pre- and postneoadjuvant chemotherapy. Although the study was retrospective, the administration of the neoadjuvant chemotherapy was according to hospital protocol and uniform; after three cycles of taxane-platinum chemotherapy an interval debulking surgery procedure was performed. Sixteen patients were eligible for analysis as pre- and post chemotherapy FFPE tissues were available (Figure 2). pVEGFR2(996) expression levels after chemotherapy were lower compared with the initial untreated biopsy (median $H$-score $=50$, range $(0-300)$ vs 300, range $(120-300) ; P<0.0001)$. For the pS6 and p4E-BP1 expression there was no significant difference found. Again, the correlation of pVEGFR2(Tyr996) and pS6, and pVEGFR2(Tyr996) and p4E-BP1 was present in lesions treated after chemotherapy $(R=0.44$, $P=0.002-R=0.27 P=0.054)$. 


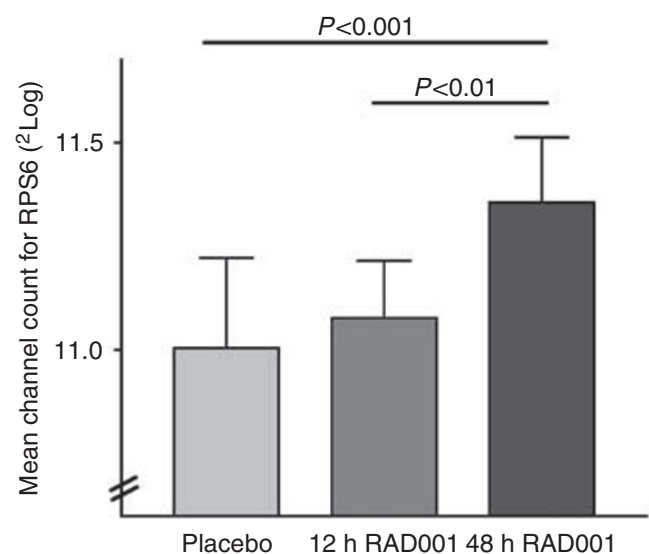

Gene expression of RPS6 after mTOR inhibition

(One-way ANOVA $P=0.0002$ )

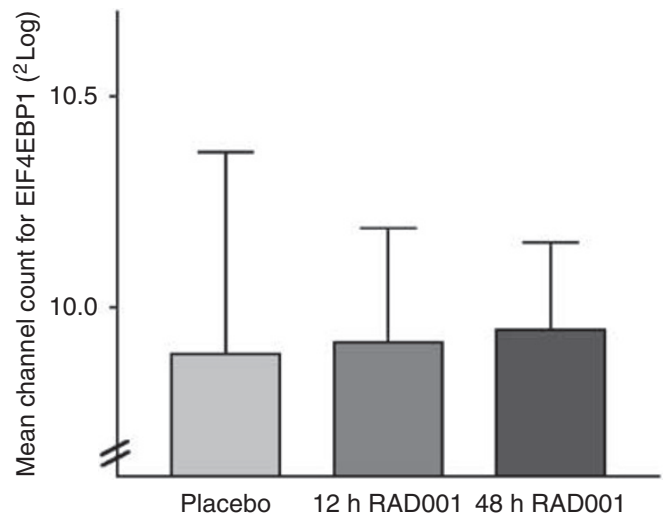

Gene expression of EIF4EBP1 after mTOR inhibition

(One-way ANOVA $P=0.95$ )

Figure 6 After $48 \mathrm{~h}$ RAD00 I administration, prostate tissue showed a significant increase of normalised gene expression for RPS6 compared with $12 \mathrm{~h}$ $\left(P<0.01-{ }^{2} \log\right.$ mean channel count increase of $\left.+0.2895 \% \mathrm{Cl}(0.08-0.48)\right)$ and placebo-treated mice $(P<0.00 \mathrm{I}-2$ log mean channel count increase of $+0.3595 \% \mathrm{Cl}(0.17-0.53))$, This effect was not observed for the expression of EIF4EBPI (n.s.)

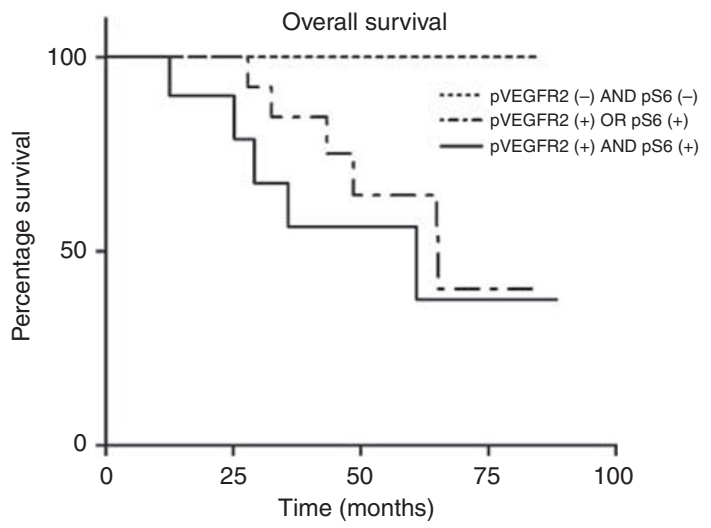

Figure 7 Kaplan-Meier survival curve ( $N=32$ patients). Baseline expression of pS6 and pVEGFR2 were dichotomised into a high $(+)$ and low (-) category using the median expression $\mathrm{H}$-score value (log-rank test, $P=0.041)$.

\section{Gene expression analysis}

Next, the observed immunohistochemical correlation of pVEGFR2 and pS6 was studied in silico at a gene expression (mRNA) level from cDNA microarrays of 24 ovarian cancers from the Erasmus MC centre (Helleman et al, 2006). Relative gene expressions were correlated between the VEGFR2 gene and the RPS6 and EIF4EBP1 genes. There was a highly significant, but negative, correlation between the VEGFR2 and RPS6 $(R=-0.73 ; P<0.0001)$, whereas there was no correlation with EIF4EBP1 (Figure 5). This negative correlation is compatible with the findings that the gene expression of S6 and its phosphorylation status is inversely regulated.

Phosphorylation of the ribosomal protein S6 is completely inhibited after mTOR inhibition, thus protein expression of pS6 is expected to decrease (Tabernero et al, 2005). In silico analyses (Affymetrix microarray data from prostate of Mus musculus treated with mTOR inhibitor RAD001) show that this downstream marker of the AKT/mTOR signalling pathway is upregulated after mTOR inhibition. A significant, apparently time dependant, increased gene expression after mTOR inhibition of the gene RPS6 could be seen, whereas there was no significant change for EIF4EBP1 (Figure 6).

\section{Survival analysis}

The expression of pS6 and VEGFR2(Tyr996) was dichotomised using the median expression as a cutoff value. Patients were divided into three groups: (1) patients with a high expression of pS6 and pVEGFR2(Tyr996) $(N=8)$, 'activated pathway', (2) patients with a combined low expression of pS6 and pVEGFR2 in the tumour $(N=9)$ and (3) the intermediate group, in which only one out of two markers had high expression $(N=15)$. A significant reduced survival was found in patients who had a combined high expression of pVEGFR(Tyr996) and pS6 (log-rank test, $P=0.041$ ) (Figure 7 ). In an exploratory multivariate analysis using Cox Proportional Hazard regression model correcting for FIGO stage and grade, this significance is retained $(P=0.048, \mathrm{HR}$ $2.90,95 \%$ CI $(1.01-7.97))$ and FIGO staging was also found to be a independent prognostic factor $(P=0.050, \mathrm{HR} 1.99,95 \% \mathrm{CI}$ $(0.89-4.47))$.

A combined high expression of pVEGFR2(Tyr996) and pS6 was significantly associated with the presence of ascites $(87.5 \%, N=7 / 8)$, whereas in patients with a low expression of both markers, the presence of ascites was only $11.1 \%(N=1 / 9)$. In the intermediate group there was ascites in $40.0 \%(N=6 / 15)\left(P=0.002 ; \chi^{2}\right.$ test for linear by linear association).

\section{DISCUSSION}

Using human ovarian cancer samples, a correlation was observed between the activated status of the VEGFR2 and downstream markers of the AKT/mTOR signalling pathway, pS6 and p4E-BP1 protein. Especially, the correlation of pVEGFR2(Tyr996) and pS6 was found to be the most noteworthy correlation between VEGF-A signalling and AKT/mTOR signalling. For these two markers, besides the $H$-score correlations that were observed, a correlation could also be seen in heterogenic staining areas of some tumour samples, that is, places where pVEGFR2-positive cells also clearly stained more positive for pS6 (Figure 3). Two antibodies were used in this study for pVEGFR2. Mainly, the results of pVEGFR(Tyr996) were reported because pVEGFR2(Tyr996) seems to be a better potential marker for bevacizumab efficacy (Wedam et al, 2006). Similarly, for markers of the AKT/mTOR signalling pathway two antibodies were selected also, with a preference for pS6, as there is evidence that S6 is more specifically regulated by mTOR (Tabernero et al, 2008). However, p4E-BP1 was also chosen because it has been shown to be a hallmark protein downstream of 
mTOR that is associated with grade and survival in ovarian cancer as well as in breast cancer (Castellvi et al, 2006; Rojo et al, 2007).

Gene expression analysis showed that, after mTOR inhibition, the gene expression of S6 changed, whereas for 4E-BP1 it did not. Although the change fold $(<2)$ was minimal, it was an apparently time dependant, clearly significant change. Here, the negative correlation of VEGFR2 and RPS6, is compatible with the finding that the phosphorylation of S6 is inversely regulated with its gene expression. These data are therefore indirectly confirmative for the immunohistochemical findings that showed a positive correlation.

Given our current knowledge, there are many uncertainties and it is too early to rely completely on biomarkers for clinical usage. To fully appreciate the value of biomarkers, extensive translational research in considerable number of patients, are needed (Sleijfer and Wiemer, 2008).

Nevertheless, our findings strongly suggest activation of the VEGFR2 pathway in ovarian cancer. Consistent with our data, earlier, a similar study with human samples has shown the expression of the VEGFR2 to be been associated with the activation of the signal transducers and activators of transcription pathway, another intracellular signalling pathway of VEGFR2 (Chen et al, 2004). The most likely way by which this receptor is activated is through binding of its ligand, VEGF-A. Platelets are a major source of VEGF-A that is targeted by bevacizumab (Verheul et al, 2007). As the ovarian tumour cells are known to excrete the endothelial growth factor VEGF-A as well, this suggests an autocrine/paracrine growth factor function of VEGF-A in ovarian cancer through the AKT/mTOR signalling pathway. The dual expression of VEGFR/ VEGF-A in ovarian cancer, as well as in other types of cancers, suggests that the activation of tumour cell signalling pathways by VEGF-A might be an autocrine mechanism that could also be present in other tumours such as colorectal or breast carcinoma (Masood et al, 2001; Price et al, 2001; Chen et al, 2004; Fan et al, 2005; Wedam et al, 2006).

Anti-angiogenesis with agents such as bevacizumab are believed to act through blocking VEGF-A action on endothelial cells. The unseen antitumoural effects observed after bevacizumab treatment in refractory- and platinum-resistant ovarian cancer patients indicate that these responses possibly are not only caused by inhibition of angiogenesis. We hereby show that a presumed activated VEGFR2/AKT/mTOR pathway, that is endothelial cell independent, was found to be associated with significantly reduced survival. Although the study cohort was small, without searching for a cutoff value, significance could be shown. The fact that this activated pathway was associated with increasing incidence of ascites is maybe not surprising. VEGF-A, formerly know as vascular permeability factor, has been found to play an important role in the accumulation of pleural or peritoneal effusions (Senger et al, 1983; Nagy et al, 1993, 1995; Yeo et al, 1993; Masood et al, 2001). Also, in preclinical studies, the bevacizumab murine precursor was more an inhibitor of ascites formation than that it was an inhibitor of tumour growth (Mesiano et al, 1998).

The observed correlation between the VEGF-A pathway and the AKT/mTOR pathway has potential clinical importance. mTOR inhibitors gain much clinical interest as an antitumoural agent, such as in renal cell carcinoma (Hudes et al, 2007). For ovarian cancer, clinical trials are ongoing. Up to date, there is only preclinical data available to suggest that mTOR inhibition might be beneficial in epithelial ovarian cancer. Altomare et al, 2004 studied the phosphorylation of AKT on ovarian cancers in a tissue microarray and found overexpression of p-AKT on $68 \%$ of the 31 tumours. Mabuchi et al, 2007a, 2007b found that mTOR inhibition by RAD001 reduced human ovarian cancer cell proliferation, enhanced cisplatin-induced apoptosis and prolonged survival in an ovarian cancer xenograft model. They also showed that RAD001 inhibited the expression of HIF 1 alpha and VEGF-A in vitro cell lines. Interestingly, dual targeting of VEGF-A and mTOR in ovarian caner xenograft models has shown an additive, if not synergistic, antitumoural effect with survival benefit. Additionally, the combination therapy was able to reverse the accumulation of ascites, which is in agreement with our findings (Huynh et al, 2007).

Anti-VEGF treatments in ovarian cancer seem to be very active, although at this moment, the associated toxicity is worrisome. mTOR inhibitors might have the potential of avoiding these problems Taking our data into consideration, suggestive of an autocrine VEGF-A loop through the AKT/mTOR signalling pathway, this adds preclinical rationale for mTOR inhibition in the management of ovarian cancer. The results of the GOG phase II trial, which is ongoing, will reveal if temsirolimus has single-agent activity in recurrent/refractory patients.

We started a multicentre prospective study in 2006 with the aim of standardised collection of snap frozen human ovarian cancer tissues. Similar experiments will reveal if our present findings can be confirmed. We will try to further elucidate the interaction between both the pathways at a more detailed gene expression level. In any future clinical trials, we emphasise the necessity of tissue/ascites sampling for translational and biomarker studies.

In conclusion, we propose that the working mechanism of antiVEGF treatments in epithelial ovarian cancer is not only antiangiogenesis. We strongly suggest that these anti-VEGF treatments are suppressors of epithelial tumour cell growth factor acting as a surrogate AKT/mTOR signalling inhibitors on tumour cells. Thus, classifying VEGF trap or bevacizumab as anti-angiogenic agent does not represent their whole mechanism of action. Based on our findings, we recommend them as anti-VEGF compounds, at least in epithelial ovarian cancer.

\section{ACKNOWLEDGEMENTS}

We gratefully thank Stefan Sleijfer from the Erasmus MC, Rotterdam, The Netherlands for discussing, editing and correcting this manuscript.

\section{Conflict of interest}

The authors state no conflict of interest

\section{REFERENCES}

Altomare DA, Wang HQ, Skele KL, De Rienzo A, Klein-Szanto AJ, Godwin AK, Testa JR (2004) AKT and mTOR phosphorylation is frequently detected in ovarian cancer and can be targeted to disrupt ovarian tumor cell growth. Oncogene 23(34): 5853-5857

Badgwell BD, Camp ER, Feig B, Wolff RA, Eng C, Ellis LM, Cormier JN (2008) Management of bevacizumab-associated bowel perforation: a case series and review of the literature. Ann Oncol 19(3): 577-582

Burger RA (2007) Experience with bevacizumab in the management of epithelial ovarian cancer. J Clin Oncol 25(20): 2902-2908
Burger RA, Sill MW, Monk BJ, Greer BE, Sorosky JI (2007) Phase II trial of bevacizumab in persistent or recurrent epithelial ovarian cancer or primary peritoneal cancer: a Gynecologic Oncology Group Study. J Clin Oncol 25(33): 5165-5171

Cannistra SA, Matulonis UA, Penson RT, Hambleton J, Dupont J, Mackey H, Douglas J, Burger RA, Armstrong D, Wenham R, McGuire W (2007) Phase II study of bevacizumab in patients with platinumresistant ovarian cancer or peritoneal serous cancer. J Clin Oncol 25(33): $5180-5186$ 
Castellvi J, Garcia A, Rojo F, Ruiz-Marcellan C, Gil A, Baselga J, Cajal S (2006) Phosphorylated 4E binding protein 1: a hallmark of cell signaling that correlates with survival in ovarian cancer. Cancer 107(8): $1801-1811$

Chen H, Ye D, Xie X, Chen B, Lu W (2004) VEGF, VEGFRs expressions and activated STATs in ovarian epithelial carcinoma. Gynecol Oncol 94(3): $630-635$

Cobleigh MA, Langmuir VK, Sledge GW, Miller KD, Haney L, Novotny WF, Reimann JD, Vassel A (2003) A phase I/II dose-escalation trial of bevacizumab in previously treated metastatic breast cancer. Semin Oncol 30(5 Suppl 16): $117-124$

Fan F, Wey JS, McCarty MF, Belcheva A, Liu W, Bauer TW, Somcio RJ, Wu Y, Hooper A, Hicklin DJ, Ellis LM (2005) Expression and function of vascular endothelial growth factor receptor-1 on human colorectal cancer cells. Oncogene 24(16): 2647-2653

Ferrara N (2004) Vascular endothelial growth factor: basic science and clinical progress. Endocr Rev 25(4): 581-611

Ferrara N (2005) VEGF as a therapeutic target in cancer. Oncology 69(Suppl 3): $11-16$

Giantonio BJ, Catalano PJ, Meropol NJ, O'Dwyer PJ, Mitchell EP, Alberts SR, Schwartz MA, Benson III AB (2007) Bevacizumab in combination with oxaliplatin, fluorouracil, and leucovorin (FOLFOX4) for previously treated metastatic colorectal cancer: results from the Eastern Cooperative Oncology Group Study E3200. J Clin Oncol 25(12): $1539-1544$

Helleman J, Jansen MP, Span PN, van Staveren IL, Massuger LF, Meijer-van Gelder ME, Sweep FC, Ewing PC, van der Burg ME, Stoter G, Nooter K, Berns EM (2006) Molecular profiling of platinum resistant ovarian cancer. Int J Cancer 118(8): 1963 - 1971

Hudes G, Carducci M, Tomczak P, Dutcher J, Figlin R, Kapoor A, Staroslawska E, Sosman J, McDermott D, Bodrogi I, Kovacevic Z, Lesovoy V, Schmidt-Wolf IG, Barbarash O, Gokmen E, O'Toole T, Lustgarten S, Moore L, Motzer RJ (2007) Temsirolimus, interferon alfa, or both for advanced renal-cell carcinoma. N Engl J Med 356(22): $2271-2281$

Huynh H, Teo CC, Soo KC (2007) Bevacizumab and rapamycin inhibit tumor growth in peritoneal model of human ovarian cancer. Mol Cancer Ther 6(11): 2959-2966

Inan S, Vatansever S, Celik-Ozenci C, Sanci M, Dicle N, Demir R (2006) Immunolocalizations of VEGF, its receptors flt-1, KDR and TGF-beta's in epithelial ovarian tumors. Histol Histopathol 21(10): 1055-1064

Jemal A, Siegel R, Ward E, Hao Y, Xu J, Murray T, Thun MJ (2008) Cancer statistics, 2008. CA Cancer J Clin 58(2): 71-96

Kelland LR (2005) Emerging drugs for ovarian cancer. Expert Opin Emerg Drugs 10(2): $413-424$

Mabuchi S, Altomare DA, Cheung M, Zhang L, Poulikakos PI, Hensley HH, Schilder RJ, Ozols RF, Testa JR (2007a) RAD001 inhibits human ovarian cancer cell proliferation, enhances cisplatin-induced apoptosis, and prolongs survival in an ovarian cancer model. Clin Cancer Res 13(14): $4261-4270$

Mabuchi S, Altomare DA, Connolly DC, Klein-Szanto A, Litwin S, Hoelzle MK, Hensley HH, Hamilton TC, Testa JR (2007b) RAD001 (Everolimus) delays tumor onset and progression in a transgenic mouse model of ovarian cancer. Cancer Res 67(6): 2408-2413

Martin L, Schilder R (2007) Novel approaches in advancing the treatment of epithelial ovarian cancer: the role of angiogenesis inhibition. J Clin Oncol 25(20): 2894-2901

Masood R, Cai J, Zheng T, Smith DL, Hinton DR, Gill PS (2001) Vascular endothelial growth factor (VEGF) is an autocrine growth factor for VEGF receptor-positive human tumors. Blood 98(6): 1904-1913

Mesiano S, Ferrara N, Jaffe RB (1998) Role of vascular endothelial growth factor in ovarian cancer: inhibition of ascites formation by immunoneutralization. Am J Pathol 153(4): 1249-1256
Nagy JA, Herzberg KT, Dvorak JM, Dvorak HF (1993) Pathogenesis of malignant ascites formation: initiating events that lead to fluid accumulation. Cancer Res 53(11): 2631-2643

Nagy JA, Morgan ES, Herzberg KT, Manseau EJ, Dvorak AM, Dvorak HF (1995) Pathogenesis of ascites tumor growth: angiogenesis, vascular remodeling, and stroma formation in the peritoneal lining. Cancer Res 55(2): $376-385$

Price DJ, Miralem T, Jiang S, Steinberg R, Avraham H (2001) Role of vascular endothelial growth factor in the stimulation of cellular invasion and signaling of breast cancer cells. Cell Growth Differ 12(3): 129-135

Rojo F, Najera L, Lirola J, Jimenez J, Guzman M, Sabadell MD, Baselga J, Cajal S (2007) 4E-binding protein 1, a cell signaling hallmark in breast cancer that correlates with pathologic grade and prognosis. Clin Cancer Res 13(1): $81-89$

Senger DR, Galli SJ, Dvorak AM, Perruzzi CA, Harvey VS, Dvorak HF (1983) Tumor cells secrete a vascular permeability factor that promotes accumulation of ascites fluid. Science 219(4587): 983-985

Sleijfer S, Wiemer E (2008) Dose selection in phase I studies: why we should always go for the top. J Clin Oncol 26(10): 1576-1578

Stewart M, Turley H, Cook N, Pezzella F, Pillai G, Ogilvie D, Cartlidge S, Paterson D, Copley C, Kendrew J, Barnes C, Harris AL, Gatter KC (2003) The angiogenic receptor KDR is widely distributed in human tissues and tumours and relocates intracellularly on phosphorylation. An immunohistochemical study. Histopathology 43(1): 33-39

Tabernero J, Rojo F, Burris H, Casado E, Macarulla T, Jones S, Dimitrijevic S, Hazell K, Shand N, Baselga J (2005) A phase I study with tumor molecular pharmacodynamic (MPD) evaluation of dose and schedule of the oral mTOR-inhibitor everolimus (RAD001) in patients (pts) with advanced solid tumors. J Clin Oncol 23(16S): 3007

Tabernero J, Rojo F, Calvo E, Burris H, Judson I, Hazell K, Martinelli E, Cajal S, Jones S, Vidal L, Shand N, Macarulla T, Ramos FJ, Dimitrijevic S, Zoellner U, Tang P, Stumm M, Lane HA, Lebwohl D, Baselga J (2008) Doseand schedule-dependent inhibition of the mammalian target of rapamycin pathway with everolimus: a phase I tumor pharmacodynamic study in patients with advanced solid tumors. J Clin Oncol 26(10): 1603-1610

Van den Eynden GG, Van der Auwera I, Van Laere SJ, Colpaert CG, Turley H, Harris AL, van Dam P, Dirix LY, Vermeulen PB, van Marck EA (2005) Angiogenesis and hypoxia in lymph node metastases is predicted by the angiogenesis and hypoxia in the primary tumour in patients with breast cancer. Br J Cancer 93(10): 1128-1136

Van den Eynden GG, Vandenberghe MK, van Dam PJ, Colpaert CG, van Dam P, Dirix LY, Vermeulen PB, van Marck EA (2007) Increased sentinel lymph node lymphangiogenesis is associated with nonsentinel axillary lymph node involvement in breast cancer patients with a positive sentinel node. Clin Cancer Res 13(18 Pt 1): 5391-5397

Verheul HM, Lolkema MP, Qian DZ, Hilkes YH, Liapi E, Akkerman JW, Pili R, Voest EE (2007) Platelets take up the monoclonal antibody bevacizumab. Clin Cancer Res 13(18 Pt 1): $5341-5347$

Wedam SB, Low JA, Yang SX, Chow CK, Choyke P, Danforth D, Hewitt SM, Berman A, Steinberg SM, Liewehr DJ, Plehn J, Doshi A, Thomasson D, McCarthy N, Koeppen H, Sherman M, Zujewski J, Camphausen K, Chen H, Swain SM (2006) Antiangiogenic and antitumor effects of bevacizumab in patients with inflammatory and locally advanced breast cancer. J Clin Oncol 24(5): 769-777

Yang JC, Haworth L, Sherry RM, Hwu P, Schwartzentruber DJ, Topalian SL, Steinberg SM, Chen HX, Rosenberg SA (2003) A randomized trial of bevacizumab, an anti-vascular endothelial growth factor antibody, for metastatic renal cancer. $N$ Engl J Med 349(5): 427-434

Yeo KT, Wang HH, Nagy JA, Sioussat TM, Ledbetter SR, Hoogewerf AJ, Zhou Y, Masse EM, Senger DR, Dvorak HF (1993) Vascular permeability factor (vascular endothelial growth factor) in guinea pig and human tumor and inflammatory effusions. Cancer Res 53(12): 2912-2918 